\title{
VIRTUAL REALITY TECHNOLOGY DEVELOPMENT IN KOREA
}

\author{
ChanJong Park \\ VR Team, Human Computing Div., CSCL, ETRI \\ Taejun, Korea
}

\begin{abstract}
Korea is constructing its information highway scheduled for completion by 2015. However, not all this highway infrastructure is part of the national strategy. The development plan includes extensive software applications for the movie, game, and multimedia industries.

At the present time the Internet can represented as the sea of information (cyber space) which can be searched for any information, new concept, or promotion over time and space. The increasing number and demands of Internet users drive the development. In particular, those demands are promoting the movement from $2 \mathrm{D}$ to $3 \mathrm{D}$ environments, as well as interactions that make the virtual, or cyber, world more closely resemble the real world.

With those efforts go an increasing interest in virtual reality (VR), and there is currently much VR research and the development of commercial VR applications taking place in Korea. In this paper I describe the technology trends, the organizations involved in VR research, and introduce the other articles in this issue of the IJVR.
\end{abstract}

\section{VR Technology Trends in Industry}

\subsection{WOOBO ELECTRONICS CO.}

The principal product of Woobo Electronics Co. is VR software for the PC supporting a stereoscopic image format. In particular, they have been successful in commercializing several products including LCD BIOS for display related to stereoscopic images, stereoscopic plug-in software supporting VRML, and stereoscopic display drivers for web browsers. They plan to develop a new version of Cyberborg game software that includes LCD shutter glasses, and export it to foreign countries this year.

\subsection{KASAN ELECTRONICS}

Kasan Electronics, well known for its VGA expertise in Korea, has developed the PC based $3 D$ Max board, a stereoscopic display card for a PC virtual reality system. Moreover, they developed a VGA card applying hyper pipeline 3D architecture and a video acceleration function to remove data 
bottlenecks. This card can provide a high quality VR environment and is optimized for an Pentium II processor platform.

\subsection{ADAM AND THE OTHER COMPANIES}

After the first appearance of Kyoko Date in Japan, there has been a boom of the virtual characters throughout the world. In Korea there have been many attempts to make virtual characters. First, a cyber singer Adam appeared (see Figure 1), followed by Lusia and Cyda. They were all introduced as singers, publishing their music albums and movie clips.

Figure 1 shows Adam [1]. These cyber characters began to lose popularity as Kyoko Date did, however, because of limitations including artificial appearance, difficulty in producing media, and live performances. Although they failed to make a big hit, they ushered in an era of numerous cyber characters. For instance, Zzaloo is the cyber baby in the video Bebe and Lion has been introduced as a virtual student of Kyunghee University. Besides them, there virtual professors, writers, journalists, and so on. As Adam, we expect new versions of Lusia and Cyda to compensate for their technical limitations [8].

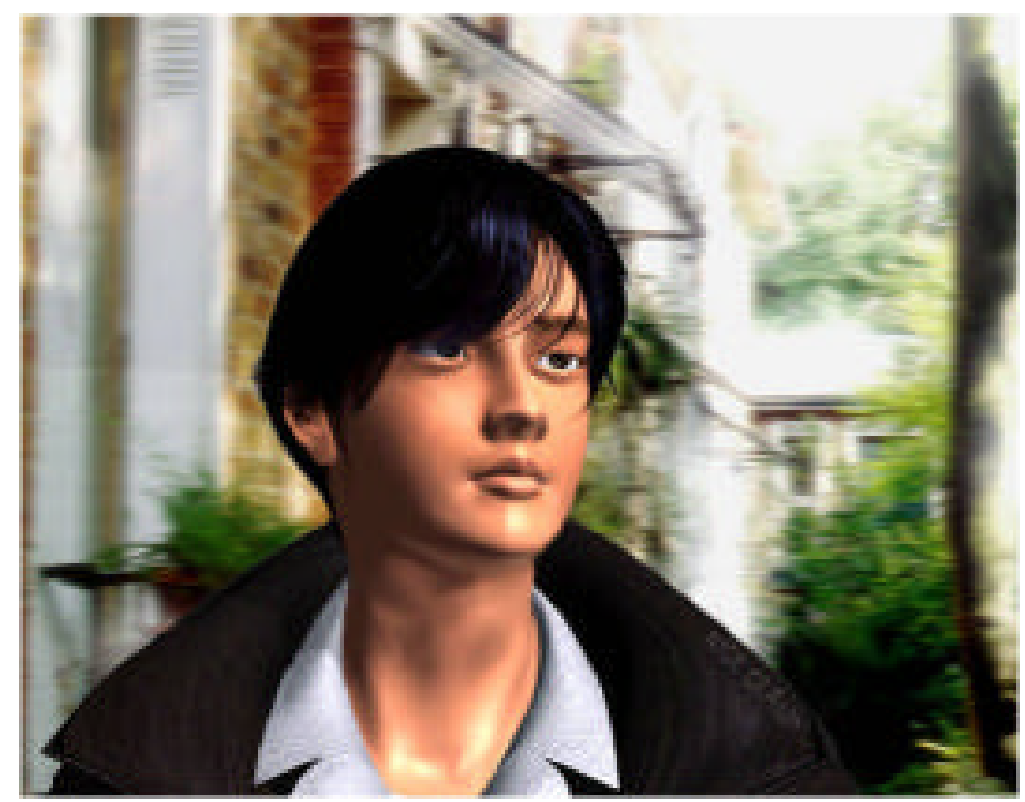

Figure 1: Adam (A Cyber Singer in Korea).

\section{VR Research at Universities}

\subsection{KAIST (KOREA ADVANCED INSTITUTE}

OF SCIENCE \& TECHNOLOGY) [2]

In KAIST, there are two groups that carry on research related to VR. One, called the Virtual Reality Group, conducts researches in areas of networked VR, distributed VR, HCI (Human-Computer Interaction), reflexive VR system, behavior modeling, physics-based animation, and facial animation. 
The main product is CVRAT (Collaborative Virtual Reality Authoring Tool) which supports multiple users. The other, called the Computer Graphics Group, does research on core algorithms and base software in computer graphics involving fractal image generation, geometric modeling, image morphing and warping, physically based modeling, shading and rendering, and solid modeler.

\subsection{COMPUTER ANIMATION AND VR LABORATORY IN SEOUL NATIONAL UNIVERSITY [3]}

Computer animation and VR applications are the focuses of this laboratory. The main research interests an efficient animation technique and its application to human animation. There is a virtual environment in their web site that can maintain numerous agents concurrently. It is developed by combining real-time rendering and animation techniques.

\subsection{VIRTUAL REALITY LABORATORY IN POHANG}

\section{UNIVERSITY OF SCIENCE \& TECHNOLOGY [4]}

The VR research in this Laboratory covers not only VR technology, but also computer graphics and real-time simulation that are the bases of VR technology. The laboratory is interested in both theory and applications of computer graphics and computer animation. The research on VR technology includes navigation in a virtual building, VRML-related technology on Internet, and behavior recognition of human participants. In the area of base technology, the laboratory studies, among other things, 3D stereo modeling \& display and the simulation of objects that can be modeled using complicated differential equations.

\subsection{COMPUTER GRAPHICS LABORATORY IN}

CHOONGANG UNIVERSITY [5]

This laboratory develops algorithms based on computer graphics involving fractal image generation, LOD (Level Of Detail), collision detection, and shadow generation. They do research on metaball rendering and radiosity natural modeling as a part of the development of an advance rendering toolkit.

\section{VR Research at Institutes}

\subsection{KIST (KOREA INSTITUTE OF}

SCIENCE AND TECHNOLOGY) [6]

There are many activities related to VR in the Division of Electronics and Information Technology at KIST. The Imaging Media Research Center pursues interactive information technology, 3D image media technology, and reality media technology. This division is well known for a virtual 
studio system that has been developed together with MBC. They also develop force feedback devices using linear motors.

\subsection{ETRI (ELECTRONICS AND TELECOMMUNICATIONS}

RESEARCH INSTITUTE) [7]

The VR Department of ETRI has seven teams that do research in the areas of computer graphics, VR, augmented reality (AR), haptic interfaces, game, and 3D sound software and technology. Since 1997, they have developed an outdoor AR system, called Magic Vision, that combines real images of a remote area with virtual geographical images in real time. This system is designed using a monitorbased AR configuration and is divided into two parts. One is the real scene acquisition system that includes a radio controlled helicopter, a wireless CCD camera, a global positioning system (GPS) attitude determination device and a wireless data communication device. Another is the processing and visualization system that includes a wireless data communication device, a PC with a video overlay card, and 3D graphics accelerators.

They have developed an authoring tool for VR, GENESIS ${ }^{\circledR}$, which runs under the World Wide Web and the PC. GENESIS allows web content developer to quickly and easily create 3D virtual space on the Internet. GENESIS offers features that can allow users to visit virtual space using an avatar of their choice. Avatars will be capable of moving between worlds created by GENESIS. It allows editing and building a virtual world imported from an external 3D model, managing virtual environment data and object information, and changing attributes of a virtual object. The software helps both the information provider and the 3D virtual environment developer by building 3D virtual environments using simple mouse clicks and menu selection without programming.

The researchers at ETRI have also developed the information system to give the blind the capability to access a computer and to recognize both text and image information in a computer. The system is composed of 3 major subsystems. The first is the tactile display for the blind to touch and to recognize the features in an image. This tactile display device is composed of the $8 \times 8$ tactile pin array. The second is a sound synthesis system for the blind to enable listening to text-based information using text to speech technology. The third is a sound recognition sub-system.

The avatar project concentrates on the avatar's behavior and interaction, and consists of the avatar creation, the motion, interaction, and network. First, the avatar creation tool enables editing of the avatar and applying anthropometric data with the intuitive and easy interface. The created avatar has 10 primitive motions, which are constructed using direct kinematics. Researchers expect that the proper utilization of the tool will give users simple navigation and communication in the virtual world. For more natural motion, they try to take advantages of the motion capture. Recently the Virtual Office Environment System (VOES) is presented as a prototype of the avatar project (see Figure 2). VOES has distributed network structure and protocol for virtual space navigation by multiple users and interaction 
through their avatars. To minimize network traffic, it takes a protocol called Virtual Space Transfer Protocol (VSTP)

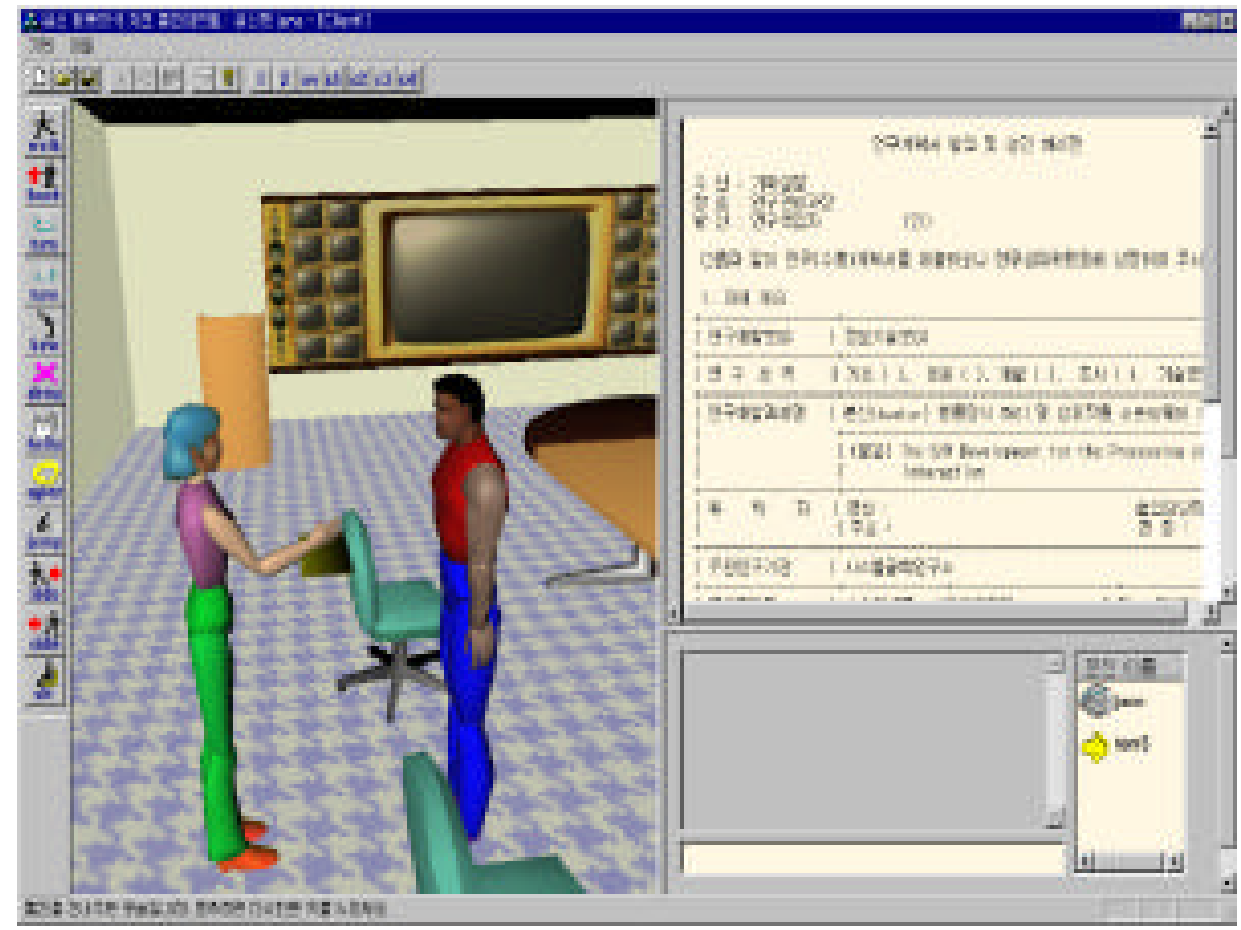

Figure 2: A snapshot of VOES

\section{Summary of the Selected Papers}

In the remainder of this issue of The International Journal of Virtual Reality there are a number of papers submitted to me by various VR researchers in Korea. I wish to thank all those authors who submitted papers for inclusion. Here, now, is an overview of those papers.

The first paper (Youngmin Lee) is concerned with the development of an augmented reality system and more specifically, an effective registration method, which is considered as the critical problem in augmented reality systems. He suggests the method for registering a virtual object in a real scene by the distortion removal algorithm for wide-angle cameras and the construction of affine coordinates from a reference object using an interactive method. It contributes to an augmented reality system for wide range with static and dynamic registration capacities, and will be a core algorithm for see-through and non-see-through HMDs.

The second paper (Hyun Suk Kim) describes the effective data structure for virtual environments. He is emphasizing the control capability of dynamic changes for temporal relationships between objects in the scene. For this purpose, he is introducing a file format, called SpanGraph, as an extension of VRML. He then applies it to the commercial broadcasting system as a virtual studio.

The third paper (Jihun Park) contributes to the control of the motion for the animation of an articulated rigid body. He introduces an extension of the generalized coordinate transformation 
technique and shows a new technique for inverse dynamic simulation of both closed and open loop systems. It will be useful for motion control of articulated objects at the kinematic level.

The fourth paper (Un-Jae Sung) describes the design of a 3-D layout system with multiple users. The main focus of this paper is the optimal responsiveness in virtual environment by a new multicastbased and optimized concurrency control mechanism. It is not new concept, but he insists on better awareness and less confusion in case of conflicts caused by moving an object in different direction.

The next four papers are published only as extended abstracts in the printed edition of this journal. The full papers are included on the CD-ROM edition.

Jinsung Choi's paper describes the VR authoring tool for multiple users. It consists of a general description of the client-server model, but also contributes to managing the shared virtual environment.

Myeong Won Lee's paper is concerned with interactive and collaborative VRML editing. The system described is too slow to use as useful tool, but it contributes as a running VRML site.

Byeong-Seok Shin's paper shows a fascinating application field of VR. The medical applications of VR are very important in diagnosis, surgery simulation, and training. The volume rendering is an interesting issue in the field of computer graphics as well.

Kyung-Ha Min's paper deals the animation technique of a virtual human or avatar. The virtual human is made by polygon mesh as skin data. The polygons of a joint area in a virtual human are not typically deformed in motion; this paper suggests a method for the deformation of skin.

\section{Conclusion}

The VR industry is immature and there are many social and ethical problems associated with the technology. However, it is not unreasonable to assume that VR will be commonplace in the future. Korean researchers are making efforts to develop new VR software and drive the VR industry in Korea. The projects and research emphasize the development of haptic interfaces, 3D VR games, and SFX technology.

\section{Acknowledgements}

I would like to thank some experts in Korea: Dr. Sung-Yong Shin (Prof. of KAIST), Dr. MyungSoo Kim (Prof. of POSTECH), Dr. Ji-In Kim (Prof. of KeonKuk Univ.), Dr. Kyung Hyun Yoon (Prof. of ChoongAng Univ.) and Dr. Choong-Whan Lee (ETRI), who reviewed and provided helpful comments on this paper. This work would not have been possible without the generous and often longterm support by them. 


\section{REFERENCES}

[1] Adam Soft, http://www.adam.co.kr/ADAM

[2] KAIST, VR Group, http://vr.kaist.ac.kr

[3] Seoul National University, CGVR Lab., http://graphics.snu.ac.kr

[4] POSTECH, http://www.cgvr.postech.ac.kr

[5] ChoongAng University, CG Lab., http://graphics.cse.cau.ac.kr

[6] KIST, http://www.kist.re.kr/98research-korean/B/54.htm

[7] ETRI, VR Department, http://human.etri.re.kr/vrt

[8] E. T. Lee, C. J. Park, and J. J. Choi, "Research Activities for Cyber Characters in Korea", To appeared in AIT'99 Taipei Taiwan.

\section{BIOGRAPHY}

Chan Jong Park is the head of the laboratory for VR at Electronics and Telecommunications Research Institute (ETRI) in South Korea. His background is in computer graphics, robotics, digital fonts, and VR. Projects that have been performed in his lab include avatars, augmented reality, authoring tools, games, and haptic systems. His recent research has been in the areas of avatar motion generation and high-level control using the motion capture data. He is a member of ACM, IEEE and KISS, KIPS of Korea, and VRSJ of Japan.

\section{Author Contact Information:}

Chan Jong Park

Header, Virtual Reality Team, ETRI

161 Kajung-dong, Yusong-gu, Taejon, KOREA

Phone: +82-42-860-1050

Fax: +82-42-860-1051

Email: cjpark@etri.re.kr (or click on mailto:cjpark@etri.re.kr while online)

Home Page: http://human.etri.re.kr/vrt/ 